Geometry 85 Topology

Volume 7 (2003) 321-328

Published: 19 May 2003

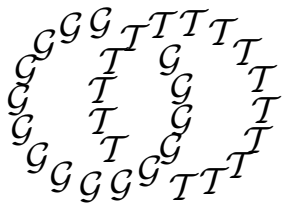

\title{
A very short proof of Forester's rigidity result
}

\author{
VINCENT GUIRARDEL \\ Laboratoire E. Picard, UMR 5580, Bâtiment 1R2 \\ Université Paul Sabatier, 118 rte de Narbonne \\ 31062 Toulouse cedex 4, France \\ Email: guirardel@picard.ups-tlse.fr
}

\begin{abstract}
The deformation space of a simplicial $G$-tree $T$ is the set of $G$-trees which can be obtained from $T$ by some collapse and expansion moves, or equivalently, which have the same elliptic subgroups as $T$. We give a short proof of a rigidity result by Forester which gives a sufficient condition for a deformation space to contain an $\operatorname{Aut}(G)$-invariant $G$-tree. This gives a sufficient condition for a JSJ splitting to be invariant under automorphisms of $G$. More precisely, the theorem claims that a deformation space contains at most one strongly slidefree $G$-tree, where strongly slide-free means the following: whenever two edges $e_{1}, e_{2}$ incident on a same vertex $v$ are such that $G_{e_{1}} \subset G_{e_{2}}$, then $e_{1}$ and $e_{2}$ are in the same orbit under $G_{v}$.
\end{abstract}

AMS Classification numbers Primary: 20E08

Secondary: $57 \mathrm{M} 07,20 \mathrm{~F} 65$

Keywords: Tree, graph of groups, folding, group of automorphisms

Proposed: Walter Neumann

Seconded: Cameron Gordon, David Gabai
Received: 24 January 2003

Revised: 11 April 2003

(c) Geometry $\mathcal{G}$ Topology $\mathcal{P}$ ublications 
In [5], Forester introduced the notion of deformation for simplicial trees with a cocompact action of a group $G$, or equivalently, for splittings of $G$ as a finite graph of groups. A deformation consists in a sequence of collapse and expansion moves in the following sense: a collapse move consists in replacing an edge in a graph of groups corresponding to an amalgamated product $A *_{C} C$ by a vertex with vertex group $A$, and an expansion move is the inverse operation.

Remember that a subgroup of $G$ is elliptic in a $G$-tree $T$ if it fixes a point in $T$. Forester proves that two cocompact simplicial $G$-trees can be deformed into one another if and only if they have the same elliptic subgroups [5, Theorem 1.1]. In terms of the geometric realization of the trees, this can also be reformulated by saying that two $G$-trees $T$ and $T^{\prime}$ can be deformed into one another if and only if there is an equivariant continuous map from $T$ to $T^{\prime}$ and one from $T^{\prime}$ to $T$.

This notion of deformation is interesting because the various JSJ splittings introduced by Rips-Sela, Dunwoody-Sageev, Fujiwara-Papasoglu [12, 3, 6] are unique up to deformation [4]. On the other hand, the JSJ splittings introduced by Bowditch for one-ended hyperbolic groups and by Scott-Swarup for finitely presented groups are really unique, up to $G$-equivariant isomorphism of trees $[2,13]$. In particular, $\operatorname{Aut}(G)$ acts naturally by isometries on the corresponding simplicial tree, or equivalently, any outer automorphism of $G$ is induced by an automorphism of the corresponding graph of groups. Therefore, this allows one to understand the automorphism group of $G$ by understanding the automorphisms of the JSJ splitting (see $[1,10]$ ).

Forester's rigidity theorem gives a sufficient condition for the existence of a canonical point in a deformation space and hence gives a criterion for a JSJ splitting à la Rips-Sela, Dunwoody-Sageev or Fujiwara-Papasoglu to be invariant under $\operatorname{Aut}(G)$.

In the sequel, we assume that all actions are without inversions (ie, no element exchanges the two endpoints of an edge) since one can get rid of inversions by taking the first barycentric subdivision of $T$. We will also assume that the actions are minimal, ie, with no proper invariant subtree. Note that if $T$ is not assumed to be minimal, but if at least one element of $G$ is not elliptic, then $T$ contains a unique minimal invariant subtree. We denote by $G_{v}$ (resp. by $G_{e}$ ) the stabilizer of a vertex $v$ (resp. of an edge $e$ ).

Definition (Strongly slide-free, reduced $G$-tree) A $G$-tree is strongly slidefree if it satifies the following condition: if two edges $e_{1}, e_{2}$ having a common vertex $v$ are such that $G_{e_{1}} \subset G_{e_{2}}$, then $e_{1}$ and $e_{2}$ are in the same orbit under $G_{v}$. 
A $G$-tree $T$ is reduced if one cannot perform a collapse on $T$, ie, if for each edge $e$ incident on some vertex $v$ such that $G_{e}=G_{v}$, then the two endpoints of $e$ are in the same orbit.

Note that in a minimal strongly slide-free $G$-tree, no vertex stabilizer can fix an edge, thus a minimal strongly slide-free splitting is itself reduced. In the following result, the deformation space of a $G$-tree $T$, is the set of all $G$-trees $T^{\prime}$ which can be deformed into $T$.

Rigidity Theorem (Forester [5, Corollary 1.3]) There is at most one strongly slide-free minimal $G$-tree in each deformation space.

More precisely, let $T, T^{\prime}$ be two minimal simplicial $G$-trees which have the same elliptic subgroups. Assume that $T$ is strongly slide-free and that $T^{\prime}$ is reduced. Then there is a $G$-equivariant isomorphism between $T$ and $T^{\prime}$ (and the isomorphism is unique).

Corollary (Forester [4]) If a group $G$ has a JSJ splitting which is strongly slide-free, then this splitting is $\operatorname{Aut}(G)$-invariant.

In particular, the action of $\operatorname{Aut}(G)$ on the Bass-Serre tree of the JSJ splitting provides a splitting of $\operatorname{Aut}(G)$ as a graph of groups.

This result extends an earlier result by Gilbert-Howie-Metaftsis-Raptis and Pettet essentially claiming that a deformation space contains at most one minimal strongly slide-free $G$-tree satisfying the additional assumption that if two adjacent edges have nested stabilizers, then these stabilizers coincide [7, 11]. This result is in turn an extension of a result by Karrass-Pietrowski-Solitar applying to amalgamated products [9].

A similar result in a different situation is proved in [8]: it is shown that in each deformation space, if there is a $G$-tree with cyclic edge stabilizers which is acylindrical, then the deformation space contains a 2 -acylindrical $G$-tree with cyclic edge stabilizers, and the set of such 2 -acylindrical $G$-trees is a simplex. This gives a way to produce an $\operatorname{Aut}(G)$-invariant JSJ-splitting for torsion free commutative transitive groups.

The proof given in [5] is quite long and involved. The goal of this note is to give a very short alternative proof, in the spirit of the proof in [7]. 


\section{Definitions}

We recall shortly a few definitions and elementary properties. Consider a $G^{-}$ tree $T$. Given a vertex $v \in V(T)$ and an edge $e \in E(T)$, we will denote by $G_{v}$ and $G_{e}$ their stabilizer. If an element $\gamma$ has a fix point in $T, \gamma$ is called elliptic, and $\gamma$ is called hyperbolic otherwise. Similarly, we say that a subgroup $H<G$ is elliptic if it fixes a point in $T$. Given an elliptic element $\gamma \in G$, the fix set Fix $\gamma$ of $\gamma$ is a subtree of $T$ (and the same of course holds for a subgroup). Serre's Lemma claims that if $\operatorname{Fix} \gamma \cap \operatorname{Fix} \gamma^{\prime}=\emptyset$, then $\gamma \gamma^{\prime}$ is hyperbolic [14, Corollary 1, section 6.5].

Given two disjoint subtrees $A, B \subset T$, the bridge between $A$ and $B$ is the smallest arc joining $A$ to $B$ : it is the arc $[a, b]$ such that $a \in A, b \in B$, and any arc joining a point of $A$ to a point of $B$ contains $[a, b]$. The projection $p(x)$ of $x$ on $A$ is the closest point to $x$ in $A$; if $x \notin A,[p(x), x]$ is the bridge between $A$ and $\{x\}$.

We will often blur the distinction between $T$ and its geometric realization, thus identifying the edge $e$ with endpoints $a, b$ to an homeomorphic copy $[a, b]$ of the interval $[0,1]$ in $\mathbb{R}$, while $\stackrel{\circ}{e}$ will represent the open segment $(a, b)=[a, b] \backslash\{a, b\}$. Note that if an element (or a subgroup) of $G$ fixes a point in $T$, then it fixes a vertex of $T$ and the fixed subtree of an element (or a subgroup) is a simplicial subtree (this uses the absence of inversion).

\section{Proof of Forester's rigidity Theorem}

Proof of the rigidity Theorem In a minimal strongly slide-free $G$-tree, no vertex stabilizer can fix an edge. In particular, vertex stabilizers of $T$ fix no more than one vertex; thus vertex stabilizers are characterized as maximal elliptic subgroups of $G$.

Let's now define a $G$-equivariant map $f: T \rightarrow T^{\prime}$. For each vertex $v \in V(T)$, choose equivariantly a vertex $f(v) \in V\left(T^{\prime}\right)$ fixed by $G_{v}$, and extend $f$ linearly and equivariantly on edges. First, the restriction of $f$ to $V(T)$ is injective: if $f(u)=f(v)$, then $\left\langle G_{u}, G_{v}\right\rangle$ is elliptic in $T^{\prime}$, hence it is also elliptic in $T$. Since vertex stabilizers of $T$ are maximal elliptic, one gets $G_{u}=G_{v}=\left\langle G_{u}, G_{v}\right\rangle$, so $u=v$. Note that this implies that the image of every edge of $T$ is a nondegenerate arc in $T^{\prime}$.

We will prove that $f$ is an isomorphism. Since $T^{\prime}$ minimal, $f$ is onto (as a topological map: some vertices of $T^{\prime}$ may have no preimage in $V(T)$ ). 
The strongly-slide free condition gives the following fact (see [7]):

Lemma 2.1 Assume that $e_{1}, e_{2} \in E(T)$ are two edges sharing a common vertex $v$ and that $f\left(e_{1}\right) \cap f\left(e_{2}\right)$ is not reduced to one point. Then $e_{1}$ and $e_{2}$ are in the same $G_{v}$-orbit and $f\left(e_{1}\right) \cap f\left(e_{2}\right)$ is strictly contained in $f\left(e_{1}\right)$ (resp. in $\left.f\left(e_{2}\right)\right)$.

Remark The lemma implies that $f\left(e_{1}\right)$ and $f\left(e_{2}\right)$ have the same length.

Proof Consider the group $H=\left\langle G_{e_{1}}, G_{e_{2}}\right\rangle<G_{v}$.

First assume that $H$ fixes only $v$ and argue towards a contradiction. Consider the vertex $w^{\prime}$ at distance 1 from $f(v)$ on $f\left(e_{1}\right) \cap f\left(e_{2}\right)$. Since $G_{w^{\prime}}$ fixes a vertex in $T$, and since $H \subset G_{w^{\prime}}, G_{w^{\prime}}$ fixes $v$ (and only $v$ ). Therefore, $G_{w^{\prime}} \subset G_{v} \subset$ $G_{f(v)}$. Since $T^{\prime}$ is reduced, $f(v)$ and $w^{\prime}$ are in the same orbit, hence $w^{\prime}$ has a preimage $w$ in the orbit of $v(w$ is thus a vertex of $T)$. Now $G_{w} \subset G_{w^{\prime}} \subset G_{v}$, hence $w=v$ by maximality of vertex stabilizers, contradicting $f(w)=w^{\prime} \neq$ $f(v)$.

Thus $H$ fixes a vertex different from $v$. Since $H$ also fixes $v, H$ fixes an edge $e_{3}$ incident on $v$. Since $G_{e_{1}}, G_{e_{2}} \subset G_{e_{3}}$, the strongly-slide free condition says that $e_{1}, e_{2}, e_{3}$ are in the same $G_{v}$-orbit.

Finally, if one had $f\left(e_{1}\right) \cap f\left(e_{2}\right)=f\left(e_{1}\right)$, then one would have $f\left(e_{1}\right)=f\left(e_{2}\right)$ since those two arcs have the same length (they are in the same orbit), and $f$ would identify two vertices, a contradiction.

Lemma 2.2 Assume that $e_{1}, e_{2}, e_{3}$ are three consecutive edges in $T$, then $f\left(e_{1}\right) \cap f\left(e_{2}\right) \cap f\left(e_{3}\right)=\emptyset$.

Proof Denote by $v_{1}$ the common vertex of $e_{1}$ and $e_{2}$, and by $v_{2}$ the common vertex of $e_{2}$ and $e_{3}$ (note that $v_{2} \neq v_{1}$ ). Assume that $f\left(e_{1}\right) \cap f\left(e_{2}\right) \cap f\left(e_{3}\right)$ contains a point $p^{\prime}$. Then $f\left(e_{1}\right)$ must meet $f\left(e_{2}\right)$ in more than one point since otherwise, $f\left(e_{2}\right)$ would be contained in $f\left(e_{3}\right)$, a contradiction. By the previous lemma, there is an element $\gamma_{1} \in G_{v_{1}}$ sending $e_{1}$ on $e_{2}$, so $\gamma_{1}$ fixes pointwise $f\left(e_{1}\right) \cap f\left(e_{2}\right)$, hence $\gamma_{1}$ fixes $p^{\prime}$. Similarly, there is an element $\gamma_{2} \in G_{v_{2}}$ sending $e_{2}$ on $e_{3}$, and which fixes $p^{\prime}$.

Now $\gamma_{1} \gamma_{2}$ is elliptic in $T^{\prime}$ (it fixes $p^{\prime}$ ) and is hyperbolic in $T$ by Serre's Lemma since Fix $\gamma_{1} \cap \operatorname{Fix} \gamma_{2}$ is empty in $T$ because neither $\gamma_{1}$ nor $\gamma_{2}$ fix $e_{2}$. This is a contradiction. 
The following lemma will say that the image under $f$ of a non-backtracking path $v_{0}, \ldots, v_{n}$ cannot backtrack too much.

Lemma 2.3 (Backtracking lemma) Consider a sequence of vertices $u_{0}, \ldots, u_{n}$ in a tree $T$ such that

(1) $u_{i} \neq u_{i+1}$ for all $i \in\{0, \ldots, n-1\}$;

(2) $\left[u_{i-1}, u_{i}\right] \cap\left[u_{i}, u_{i+1}\right]$ is strictly contained in $\left[u_{i-1}, u_{i}\right]$ and in $\left[u_{i}, u_{i+1}\right]$ for each $i \in\{1, \ldots, n-1\}$;

(3) $\left[u_{i-1}, u_{i}\right] \cap\left[u_{i}, u_{i+1}\right] \cap\left[u_{i+1}, u_{i+2}\right]=\emptyset$ for each $i \in\{1, \ldots, n-2\}$.

Then for $|j-i| \geq 2,\left[u_{i-1}, u_{i}\right] \cap\left[u_{j-1}, u_{j}\right]=\emptyset$.

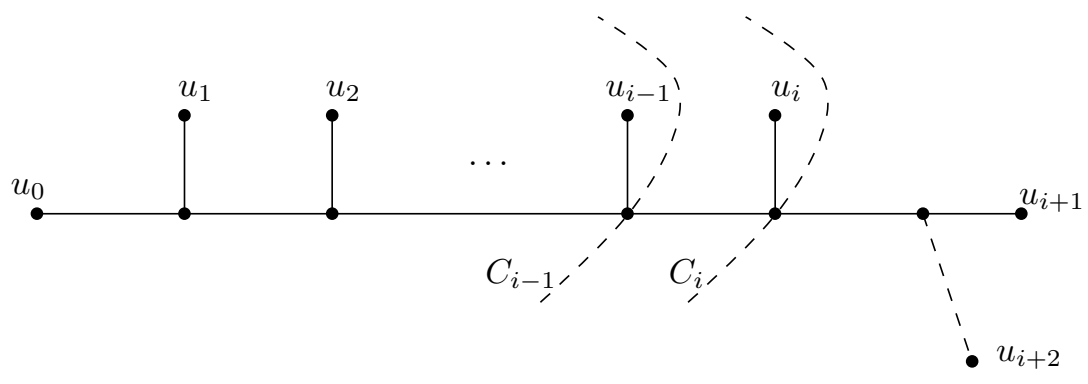

Figure 1: Backtracking lemma

Proof Let $C_{i}$ be the convex hull of $\left\{u_{0}, \ldots, u_{i}\right\}$. We prove by induction the following property for $1 \leq i \leq n-1$ :

$$
\left(P_{i}\right): \quad u_{i+1} \notin C_{i} \text { and }\left[u_{i}, u_{i+1}\right] \cap C_{i-1}=\emptyset .
$$

The lemma will then follow immediately.

Since the property clearly holds for $i=1$, we prove $P_{i} \Rightarrow P_{i+1}$. Assume that $u_{i+2} \in C_{i+1}$. Since $u_{i+2} \notin\left[u_{i}, u_{i+1}\right]$ by hypothesis (2), $u_{i+2}$ lies in $C_{i-1}$ or in the bridge joining $\left[u_{i}, u_{i+1}\right]$ to $C_{i-1}$. Thus $\left[u_{i+2}, u_{i+1}\right]$ must meet the bridge between $\left[u_{i}, u_{i+1}\right]$ and $C_{i-1}$, hence must meet $\left[u_{i}, u_{i-1}\right]$, which contradicts hypothesis (3).

If $\left[u_{i+1}, u_{i+2}\right]$ meets $C_{i}$, then $\left[u_{i+1}, u_{i+2}\right]$ contains the projection $p$ of $u_{i+1}$ on $C_{i}$. Note that by definition $p \in\left[u_{i}, u_{i+1}\right]$, so $p \in\left[u_{i}, u_{i+1}\right] \cap\left[u_{i+1}, u_{i+2}\right]$. By (3), $p \notin\left[u_{i-1}, u_{i}\right]$. This implies that $p \in C_{i-1}$ since $p$ belongs to $C_{i}$ but not to the bridge joining $u_{i}$ to $C_{i-1}$. Hence $p \in C_{i-1} \cap\left[u_{i}, u_{i+1}\right]$, which contradicts the induction hypothesis. 
Now let's conclude the proof of the theorem. Assume that $f$ is not an isomorphism. Then there exist two edges $e_{1}, e_{2}$ incident on a common vertex $v$ such that $f\left(e_{1}\right) \cap f\left(e_{2}\right)$ contains more than one point. Denote $v^{\prime}=f(v)$, and let $w^{\prime} \neq v^{\prime}$ be the vertex at distance 1 from $v^{\prime}$ on $f\left(e_{1}\right) \cap f\left(e_{2}\right)$.

Let $w$ be a point of $T$ (vertex or not) such that $f(w)=w^{\prime}$. Denote by $v_{0}=v, v_{1}, \ldots, v_{n}$ the vertices on the smallest simplicial arc containing $[v, w]$ (in particular, $w \in\left[v_{n-1}, v_{n}\right]$, and $w=v_{n}$ if and only if $w$ is a vertex). Up to exchanging the roles of $e_{1}$ and $e_{2}$, we may assume that $\left[v_{0}, v_{n}\right]$ meets $e_{1}$ only at $v$. Define $v_{-1}$ so that $\left[v_{-1}, v_{0}\right]=e_{1}$. Thus, the vertices of the arc $\left[v_{-1}, v_{n}\right]$ are $v_{-1}, v_{0}, v_{1}, \ldots, v_{n}$. Lemma 2.1 and 2.2 say that one can apply the backtracking lemma to the sequence $u_{i}=f\left(v_{i}\right), i \in\{-1,0, \ldots, n\}$. Since $w^{\prime} \in\left[u_{-1}, u_{0}\right] \cap\left[u_{n-1}, u_{n}\right]$, one gets $n=1$. By the Lemma 2.1 , the edge $\left[v_{0}, v_{1}\right]$ is in the orbit of $e_{1}$, and $w \neq v_{1}$ cannot be a vertex.

This proves that $f^{-1}\left(w^{\prime}\right) \subset G_{v} \cdot \stackrel{\circ}{1}_{1}$. In particular, since there are no inversions on $T, G_{w^{\prime}} \subset G_{v}$, therefore $G_{w^{\prime}} \subset G_{v^{\prime}}$. Since $T^{\prime}$ is reduced, this means that $w^{\prime}$ is in the same orbit as $v^{\prime}$, which contradicts the fact that $f^{-1}\left(w^{\prime}\right)$ does not contain any vertex.

It follows that $f$ maps edges to edges, so $f$ is an isomorphism. In particular, $f$ induces a bijection from $V(T)$ onto $V\left(T^{\prime}\right)$.

The uniqueness of $f$ will then follow from the following fact: given $v \in V(T)$, there is at most one vertex $v^{\prime} \in V\left(T^{\prime}\right)$ such that $G_{v} \subset G_{v^{\prime}}$. As a matter of fact, consider $w^{\prime} \in V\left(T^{\prime}\right)$ with $G_{v} \subset G_{w^{\prime}}$ and let $w$ be a preimage of $w^{\prime}$ in $V(T)$. Then $\left\langle G_{w}, G_{v}\right\rangle \subset G_{w^{\prime}}$ so $\left\langle G_{w}, G_{v}\right\rangle$ is elliptic in $T$. Therefore, $v=w$ since vertex stabilizers of $T$ fix no more than one vertex in $T$, and $v^{\prime}=w^{\prime}$.

\section{References}

[1] Hyman Bass, Renfang Jiang, Automorphism groups of tree actions and of graphs of groups, J. Pure Appl. Algebra 112 (1996) 109-155

[2] Brian H Bowditch, Cut points and canonical splittings of hyperbolic groups, Acta Math. 180 (1998) 145-186

[3] MJ Dunwoody, ME Sageev, JSJ-splittings for finitely presented groups over slender groups, Invent. Math. 135 (1999) 25-44

[4] Max Forester, On uniqueness of JSJ decompositions of finitely generated groups, to appear in Comment. Math. Helv. http://www.maths. warwick.ac.uk/ forester/

[5] Max Forester, Deformation and rigidity of simplicial group actions on trees, Geom. Topol. 6 (2002) 219-267 
[6] Koji Fujiwara, Panos Papasoglu, JSJ decomposition of finitely presented groups and complexes of groups (1998)

http://topo.math.u-psud.fr/ papazog/preprints.html

[7] N D Gilbert, J Howie, V Metaftsis, E Raptis, Tree actions of automorphism groups, J. Group Theory 3 (2000) 213-223

[8] Vincent Guirardel, Gilbert Levitt, in preparation

[9] Abe Karrass, Alfred Pietrowski, Donald Solitar, Automorphisms of a free product with an amalgamated subgroup, from: "Contributions to group theory", Contemp. Math. 33, Amer. Math. Soc., Providence, RI (1984) 328-340

[10] Gilbert Levitt, Automorphisms of hyperbolic groups and graph of groups, arXiv:math.GR/0212088

[11] Martin R Pettet, The automorphism group of a graph product of groups, Comm. Algebra 27 (1999) 4691-4708

[12] E Rips, Z Sela, Cyclic splittings of finitely presented groups and the canonical JSJ decomposition, Ann. of Math. (2) 146 (1997) 53-109

[13] Peter Scott, Gadde A Swarup, Regular neighbourhoods and canonical decompositions for groups, see www.math.Isa.umich.edu/ pscott

[14] Jean-Pierre Serre, Arbres, amalgames, $\mathrm{SL}_{2}$, Société Mathématique de France, Paris (1977) avec un sommaire anglais, rédigé avec la collaboration de Hyman Bass, Astérisque, No. 46 